Erratum

\title{
Perturbative and non-perturbative QCD corrections to wide-angle Compton scattering
}

H.W. Huang, P. Kroll, T. Morii

Eur. Phys. J. C 23, 301-310 (2002) - DOI 10.1007/s100520100883

Published online: 8 February 2002

Erratum published online: 10 October 2003 - (C) Springer-Verlag / Società Italiana di Fisica 2003

The paper contains a sign mistake. In Eqs. (1), (25), (30) and (38) the form factor $R_{T}$ and the ratio $\kappa$ are to be replaced by $-R_{T}$ and $-\kappa$, respectively. The predictions for $A_{L L}=K_{L L}$ shown in Fig. 7, are nearly independent of this change while those for $A_{L S}=-K_{L S}$ are substantially smaller in absolute value than the results shown in Fig. 8. All other results as well as the conclusions of this paper are not affected by these corrections. 